\title{
THE HAHN DECOMPOSITION THEOREM
}

\author{
RAOUF DOSS
}

\begin{abstract}
Let $(X, \mathbb{Q}, \mu)$ be a signed measure on the $\sigma$-algebra $\mathbb{Q}$ of subsets of $X$. We give a very short proof of the Hahn decomposition theorem, namely, that $X$ can be partitioned into two subsets $P$ and $N$ such that $P$ is positive: $\mu(E)>0$ for every $E \subset P$, and $N$ is negative: $\mu(E)<0$ for every $E \subset N$.
\end{abstract}

A signed measure $\mu$ on the measurable space $(X, \mathcal{Q})$ is an extended real valued set function defined on the sets of the $\sigma$-algebra $Q$ and satisfying

(i) $\mu(\varnothing)=0$.

(ii) $\mu$ assumes, at most, one of the values $+\infty,-\infty$.

(iii) $\mu\left(\cup E_{n}\right)=\Sigma \mu\left(E_{n}\right)$ for any sequence of disjoint measurable sets $E_{n}$. Condition (iii) yields immediately the following:

(iii') If $A_{n} \searrow$ and $\mu\left(A_{1}\right) \neq \pm \infty$, then $\lim \mu\left(A_{n}\right)=\mu\left(\cap A_{n}\right)$.

In what follows we shall suppose that

(ii') $+\infty$ is the infinite value omitted by $\mu$.

We say that a set $P \in Q$ is positive (with respect to the signed measure $\mu$ ) if $\mu(E)>0$ for every measurable $E \subset P$. Similarly, $N$ is negative if $\mu(E)<0$ for every $E \subset N$.

Lemma. Every $A \in \mathbb{Q}$ with $\mu(A) \neq-\infty$ contains a positive set $P$ such that $\mu(P)>\mu(A)$.

Proof. We first show that to every $\varepsilon>0$ there corresponds $A_{\varepsilon} \subset A$ such that $\mu\left(A_{\varepsilon}\right)>\mu(A)$ and $B \subset A_{\varepsilon} \Rightarrow \mu(B)>-\varepsilon$. For otherwise, inductively, there is a sequence $B_{1} \subset A, \ldots, B_{k} \subset A \backslash\left(B_{1} \cup \cdots \cup B_{k-1}\right), \ldots$ such that $\mu\left(B_{k}\right)<-\varepsilon$. Put $B=\cup B_{k}$. Since the $B_{k}$ are disjoint, then $\mu(B)=-\infty, \mu(A \backslash B)=\mu(A)-$ $\mu(B)=+\infty$, against (ii'). Now choosing $\varepsilon_{r} \rightarrow 0, A_{\varepsilon_{n}} \searrow$ and putting $P=\cap A_{e_{n}}$ we see that $P$ is positive and by (iii') $\mu(P) \geqslant \mu(A)$.

The Hahn Decomposition Theorem. Let $\mu$ be a signed measure on the measurable space $(X, Q)$. Then $X$ can be partitioned into a positive set $P$ and a negative set $N$.

Proof. Let $s=\sup \{\mu(A): A \in \mathbb{Q}\}$. There is a sequence $P_{n}$ such that $\mu\left(P_{n}\right) \rightarrow s$ and, by the lemma, we may assume the $P_{n}$ are positive. Putting $P=\cup P_{n}$ we have $\mu(P)=s$ and $P$ is positive. But $N=X \backslash P$ is negative, for if $E \subset N$ and $\mu(E)>0$ then $\mu(P \cup E)>s$ which is impossible.

The proof is now complete.

Department of Mathematics, State University of New York, Stony Brook, New York 11794

Received by the editors February 14, 1979.

AMS (MOS) subject classifications (1970). Primary 28A10. 